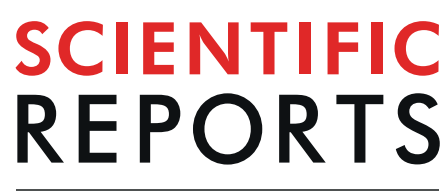

natureresearch

Corrected: Author Correction

\title{
OPEN
}

Received: 17 July 2019

\section{Thermal equation of state of ruthenium characterized by resistively heated diamond anvil}

cell

\author{
Simone Anzellini $\mathbb{1}^{1}{ }^{1}$, Daniel Errandonea $\mathbb{1}^{2}$, Claudio Cazorla $\mathbb{1}^{3}$, Simon MacLeod ${ }^{4,5}$, \\ Virginia Monteseguro ${ }^{2}$, Silvia Boccato ${ }^{6}$, Enrico Bandiello ${ }^{2}$, Daniel Diaz Anichtchenko ${ }^{2}$, \\ Catalin Popescu $\mathbb{1}^{7}$ \& Christine M. Beavers ${ }^{1}$
}

The high-pressure and high-temperature structural and chemical stability of ruthenium has been investigated via synchrotron X-ray diffraction using a resistively heated diamond anvil cell. In the present experiment, ruthenium remains stable in the hcp phase up to $150 \mathrm{GPa}$ and $960 \mathrm{~K}$. The thermal equation of state has been determined based upon the data collected following four different isotherms. A quasi-hydrostatic equation of state at ambient temperature has also been characterized up to $150 \mathrm{GPa}$. The measured equation of state and structural parameters have been compared to the results of $a b$ initio simulations performed with several exchange-correlation functionals. The agreement between theory and experiments is generally quite good. Phonon calculations were also carried out to show that hcp ruthenium is not only structurally but also dynamically stable up to extreme pressures. These calculations also allow the pressure dependence of the Raman-active $E_{2 g}$ mode and the silent $B_{1 g}$ mode of Ru to be determined.

Transition metals have always attracted the interest of the scientific community due to their unusual electronic and structural properties, originating from the dominant influence of their $d$ electrons. Often, they exhibit pronounced phonon anomalies as a result of complex Fermi-surface geometries coupled with strong electron-phonon interactions ${ }^{1,2}$. For these reasons, in the past decades big effort has been devoted to map and interpret the systematic properties of this metals at both ambient and extreme conditions of pressure and temperature $^{3}$. Ruthenium $(\mathrm{Ru})$ is a $4 \mathrm{~d}$ transition metal that belongs to the platinum $(\mathrm{Pt})$ group of the periodic table, along with rhodium $(\mathrm{Rh})$, palladium (Pd), osmium (Os), iridium (Ir) and $\mathrm{Pt}^{4}$. Unlike the other elements of the group, $\mathrm{Ru}$ presents only one electron in the outermost shell.

At ambient pressure, Ru has a hexagonal close packed structure ( $h c p$ ), melts at $2523 \mathrm{~K}$, and becomes superconductor at temperatures below $T_{c}=0.47 \mathrm{~K}$. In nature, it is generally found as a minor component in Pt ores. Ru is inert to most chemicals and it is generally used in wear-resistant electrical contacts and thick-film resistors ${ }^{4-6}$. In particular, it is often used alloyed with Pt and Pd as it increases their hardness. When alloyed with titanium (Ti), Ru improves its corrosion resistance, whereas, if alloyed with molibdenum (Mo) its $T_{c}$ changes to $10.6 \mathrm{~K}^{7}$. Finally, $\mathrm{Ru}$ is also used as a chemical catalyst ${ }^{8-10}$.

$\mathrm{Ru}$ alloys have been extensively studied at extreme conditions (experimentally and theoretically) to investigate possible ultra-hard materials ${ }^{11-13}$. 
In contrast with other $4 \mathrm{~d}$ transition metals, like Mo and rhenium $(\mathrm{Re})^{14-18}$, to the best of our knowledge, only few studies have been focused their attention on the characterization of pure Ru under extreme conditions. In particular, a study was performed in 1965 by Bucher et al. ${ }^{19}$ to characterize the effect of pressure on the superconductivity of Ru. The compression curve of Ru was experimentally measured up to $25 \mathrm{GPa}$ by Clendenen et al. ${ }^{20}$ in a pressure-cylinder apparatus ${ }^{21}$. More recently, Cynn et al. ${ }^{22}$ studied Ru up to $56 \mathrm{GPa}$ in diamond anvil cell (DAC), using argon (Ar) as pressure transmitting medium and energy-dispersive X-ray diffraction (ED-XRD) technique. In contrast with the results reported by Cynn et al., conclusions extracted from studies on iron-ruthenium alloys under high pressure suggest that a $h c p-f c c$ transition should be expected in the range of the tenths of $\mathrm{GPa}^{23}$. On the other hand, a few theoretical works have been performed on $h c p \mathrm{Ru}$. In one study, the stability field has been explored by means of elastic constant calculations ${ }^{24}$ and in a second study by first-principles calculations ${ }^{25}$. Information on the high-pressure and high-temperature behaviour of Ru is still lacking; in particular, regarding its melting curve and thermal equation of state.

In this work, the structural stability of Ru has been investigated at room-temperature, up to $150 \mathrm{GPa}$ by angular dispersive $(\mathrm{AD}) \mathrm{XRD}$, using helium as pressure medium. High-pressure $(H P)$ high-temperature $(H T)$ studies have also been performed to extend its structural and chemical characterization up to $975 \mathrm{~K}$. The experiments have been combined with first-principle calculations of the structural and lattice dynamic properties.

\section{Results}

Evolution at ambient temperature. Two different experimental runs were carried out at ambient temperature at the extreme conditions beamline (I15) of Diamond Light Source under quasi-hydrostatic conditions. Table 1 reports the obtained results. During Run1 the pressure was increased from ambient up to $97.5 \mathrm{GPa}$ with a maximum step of $2 \mathrm{GPa}$ between consecutive pressures.

During Run 2 the pressure was increased rapidly up to $92.2 \mathrm{GPa}$ and a finer pressure step was then used until reaching $151 \mathrm{GPa}$, the highest pressure covered in the present experiment. Under the present experimental conditions, Ru maintains its $h c p$ phase. Figure 1 shows the raw and the integrated AD-XRD patterns of Ru collected at the lowest and the highest pressure achieved at ambient temperature. In both cases, it is possible to observe the 100, 002, 101, 102, 110, 103, 112, 201, 004, 202 and 104 reflections belonging to the hcp structure of Ru. Few parasitic reflections coming from the Re gasket are also visible (labelled with red asterisks), this is caused by the $\mathrm{X}$-ray beam tails obtained on the first table of I15. However, the quality of the Ru XRD patterns is not affected by the presence of the signal from Re.

At ambient pressure, the raw XRD image shows a signal coming from a sample composed of multiple crystals of $\mathrm{Ru}$, and from a Re foil. At the highest pressure achieved $(151 \mathrm{GPa})$, the signal from the Re foil is similar to the one obtained at ambient pressure, whereas, the XRD of Ru shows the formation of some texturing, with small broadening in the diffraction peaks. This behavior is typically associated with the formation of micro-stress in the sample ${ }^{26}$. A Williamson-Hall analysis of our data indicates that the $\xi$ parameter ${ }^{27}$, which is a measure of peak broadening due to strain, changes from $0.5(1) \times 10^{-3}$ at ambient pressure to $0.6(1) \times 10^{-3}$ at $151 \mathrm{GPa}$, indicating that strains due to deviatoric stresses are small in our experiments up to the highest pressure. No evidence of structural transitions or distortions have been observed at $R T$ up to $151 \mathrm{GPa}$.

A qualitative analysis of the hydrostatic conditions of the sample has been performed by comparing the measured d-spacing of $\mathrm{Ru}$ at the highest pressure reached $(151 \mathrm{GPa})$ to the theoretical (hydrostatic) one calculated using the lattice parameter obtained by a refinement of the entire XRD pattern ${ }^{26}$. Table 2 shows the interplanar spacing measured at the highest pressure reached in this experiment. They deviate from the spacing calculated using the lattice parameters obtained by the refinement of the whole XRD pattern by less than $0.008 \%$. This is within the experimental error of the present experiment. Therefore, we can conclude that the non-hydrostatic effects are below the detection limit of the present measurements. That is in agreement with the quantification of the macroscopic non-hydrostatic stress on metals embedded in helium that reaches $0.5 \mathrm{GPa}$ at $150 \mathrm{GPa}^{28}$.

In Fig. 2, the obtained compression curve is reported together with the c/a evolution as a function of pressure. The corresponding bulk modulus $K_{0}$, its pressure derivative $K_{0}^{\prime}$ and the volume $V_{0}$ at ambient temperature have been determined from a least-square fit of the entire set of data to a Rydberg-Vinet ${ }^{29}$ and a third-order Birch-Murnaghan (BM3) equation of state (EoS) using the EOSFit7c software ${ }^{30}$. The obtained values are reported in Table 3. The data obtained by Cynn et al. ${ }^{22}$ with an ED-XRD DAC experiment performed using Ar as pressure transmitting medium are also reported for comparison in Fig. 2. Although their data results more scattered than the present one, both compression curves show a similar trend.

In the inset of Fig. 2, it can be seen how the c/a ratio of Ru increases with pressure, showing a tendency to approach the ideal $c / a$ value for a $h c p$ structure, thus increasing the packing efficiency of the crystal structure under compression. This phenomenon is consistent with the elasticity theory ${ }^{31}$, according to which, for a $h c p$ structure $\frac{\left(C_{33}+C_{13}\right)-\left(C_{11}+C_{12}\right)}{G}=-\partial \ln (c / a) / \partial \ln (V)$, where $G$ is the shear modulus and $C_{i i}$ are the elastic constants. According to the present DFT calculations, the first term of the previous equation is positive in the entire range of the investigated pressure domain. Therefore $\partial \ln (c / a) / \partial \ln (V)<0$. This means that the c/a ratio of $\mathrm{Ru}$ increases as the volume decreases (i.e. pressure increases) which is exactly what is experimentally observed here. In addition, apparent slope changes can also be observed in the experimentally determined c/a of Fig. 2. However, as the observed variations are smaller than the accuracy of the measurements, they can probably be caused by experimental artifacts. It is important to point here how the c/a variation of Ru from ambient pressure to $150 \mathrm{GPa}$ is only $0.6 \%$. This indicates that the compression of $\mathrm{Ru}$ occurs with little changes in its an-isotropic properties.

The c/a evolution with pressure of Ru contrasts with that of other $h c p$ metals like Os ${ }^{32}$ and $\mathrm{Re}^{17}$. On one hand, Os presents a positive slope in the c/a evolution up to $150 \mathrm{GPa}$. After this critical pressure, the slope changes into a negative one. This phenomenon is due to an Electronic Topological Transition (ETT) predicted theoretically around that pressure ${ }^{33}$. On the other hand, Re presents a negative slope in the c/a evolution between 


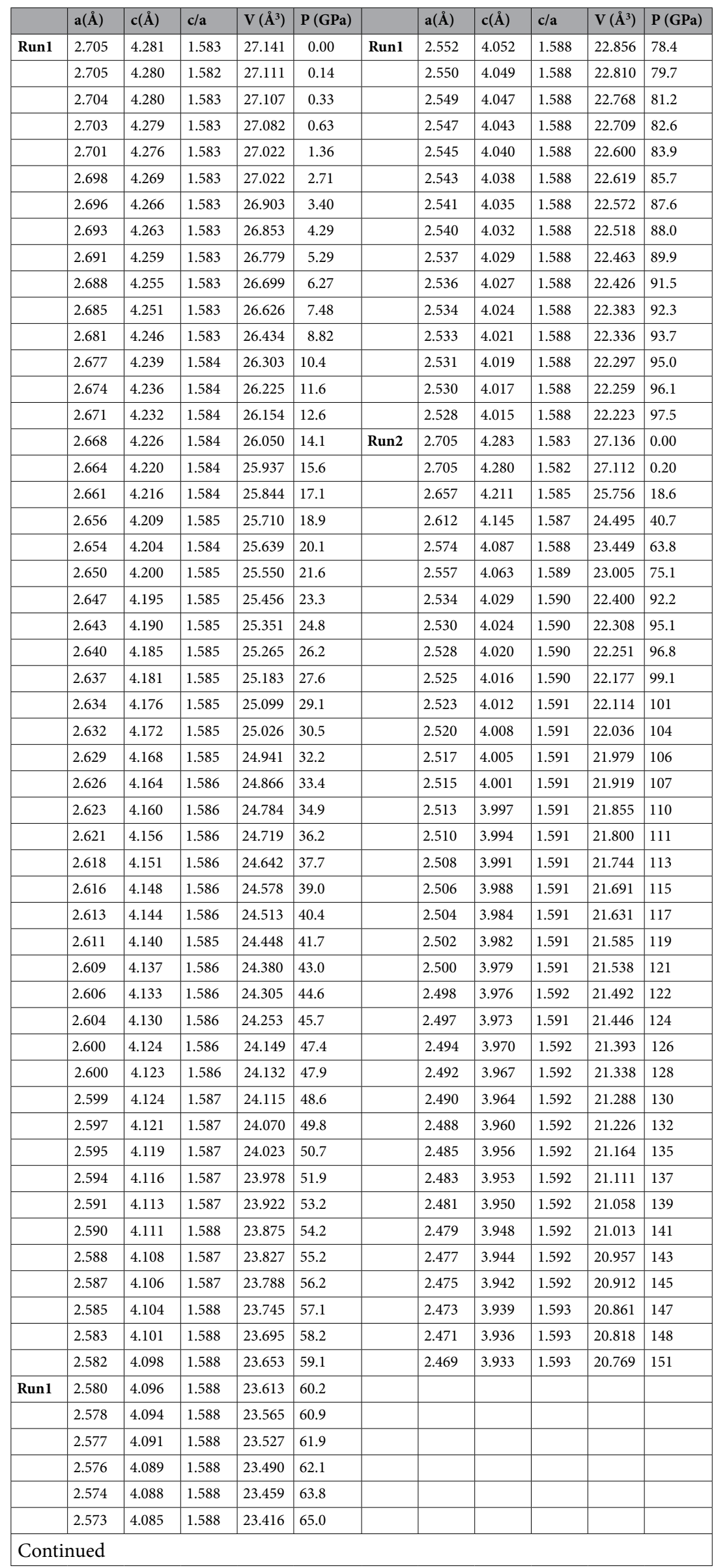




\begin{tabular}{|l|l|l|l|l|l|l|l|l|l|l|l|}
\hline & $\mathbf{a}(\AA)$ & $\mathbf{c}(\AA)$ & $\mathbf{c} / \mathbf{a}$ & $\mathbf{V}\left(\AA^{3}\right)$ & $\mathbf{P}(\mathbf{G P a})$ & & $\mathbf{a}(\AA)$ & $\mathbf{c}(\AA)$ & $\mathbf{c} / \mathbf{a}$ & $\mathbf{V}\left(\AA^{3}\right)$ & $\mathbf{P}(\mathbf{G P a})$ \\
\hline & 2.570 & 4.080 & 1.588 & 23.333 & 66.6 & & & & & & \\
\hline & 2.568 & 4.076 & 1.587 & 23.270 & 68.4 & & & & & & \\
\hline & 2.565 & 4.074 & 1.588 & 23.216 & 70.0 & & & & & & \\
\hline & 2.562 & 4.070 & 1.588 & 23.151 & 71.5 & & & & & & \\
\hline & 2.560 & 4.066 & 1.588 & 23.083 & 73.2 & & & & & & \\
\hline & 2.558 & 4.063 & 1.588 & 23.025 & 74.8 & & & & & & \\
\hline & 2.556 & 4.059 & 1.588 & 22.900 & 76.4 & & & & & & \\
\hline & 2.554 & 4.056 & 1.588 & 22.915 & 77.7 & & & & & & \\
\hline
\end{tabular}

Table 1. The unit-cell parameters of $\mathrm{Ru}$ at ambient temperature as a function of pressure. All values are obtained using $\mathrm{He}$ as pressure transmitting medium. The pressures measured with the ruby fluorescence method are all reported in GPa. The lattice parameters are reported in $\AA$. Experimental uncertainty on lattice parameters is lower than $0.003 \AA$. Uncertainty on pressure measurement increases from $0.05 \mathrm{GPa}$ at $1 \mathrm{GPa}$ to $2 \mathrm{GPa}$ at $150 \mathrm{GPa}$.

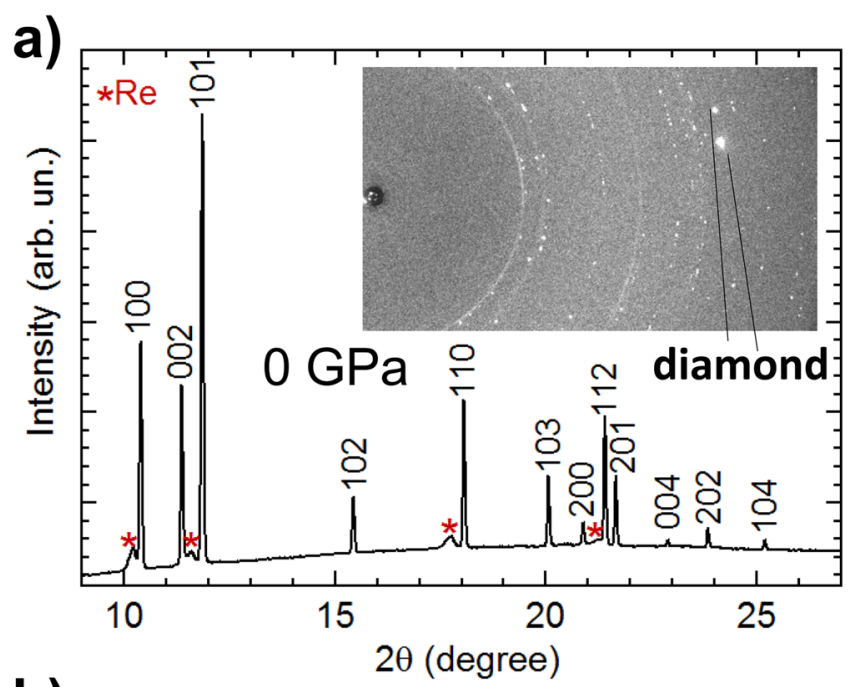

b)

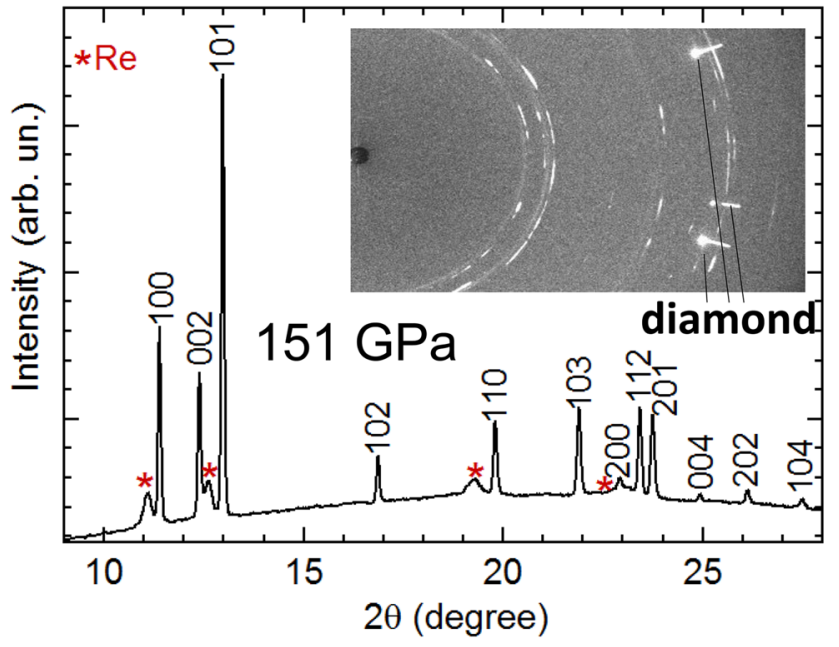

Figure 1. Integrated powder X-ray diffraction patterns of Re at low (a) and high (b) pressure. The insets show a part of the recorded raw diffraction images. The diamond contribution to the X-ray raw patterns are masked during the integration.

ambient and $150 \mathrm{GPa}^{17}$. This behaviour is probably caused by a recently discovered electronic transition called Core-Level Crossing (CLC) ${ }^{1}$. In this transition, the pressure-induced crossing of the deep $5 \mathrm{p}$ and $4 \mathrm{f}$ levels affects the valence electrons and hence the chemical bonds in the metal. This variation of chemical bonds leads to changes in the structural properties. The CLC transition cannot occurs in $\mathrm{Ru}$ as it does not have the 5p and 


\begin{tabular}{|l|l|l|l|}
\hline $\mathbf{h k l}$ & $\mathbf{d}_{\boldsymbol{m}}(\AA)$ & $\mathbf{d}_{\text {calc }}(\AA)$ & $\frac{d_{m}-d_{\text {calc }}}{d_{\text {calc }}} \%$ \\
\hline 100 & 2.1384 & 2.1384 & 0.0014 \\
\hline 002 & 1.9665 & 1.9666 & 0.0030 \\
\hline 101 & 1.8787 & 1.8787 & 0.0027 \\
\hline 102 & 1.4475 & 1.4475 & 0.0007 \\
\hline 110 & 1.2346 & 1.2346 & 0.0008 \\
\hline 103 & 1.1177 & 1.1177 & 0.0018 \\
\hline 200 & 1.0692 & 1.0692 & 0.0019 \\
\hline 112 & 1.0456 & 1.0456 & 0.0009 \\
\hline 201 & 1.0317 & 1.0318 & 0.0058 \\
\hline 004 & 0.9833 & 0.9833 & 0.0030 \\
\hline 202 & 0.9393 & 0.9394 & 0.0075 \\
\hline 104 & 0.8934 & 0.8934 & 0.0056 \\
\hline
\end{tabular}

Table 2. Measured reflections for Ru at $151 \mathrm{GPa} . h k l$ are the Miller indices of the reflection. $\mathrm{d}_{m}$ is the corresponding measured inter-planar distance measured by individual peak fitting. dcalc is the inter-planar distance calculated by a fit of the whole diffraction pattern.

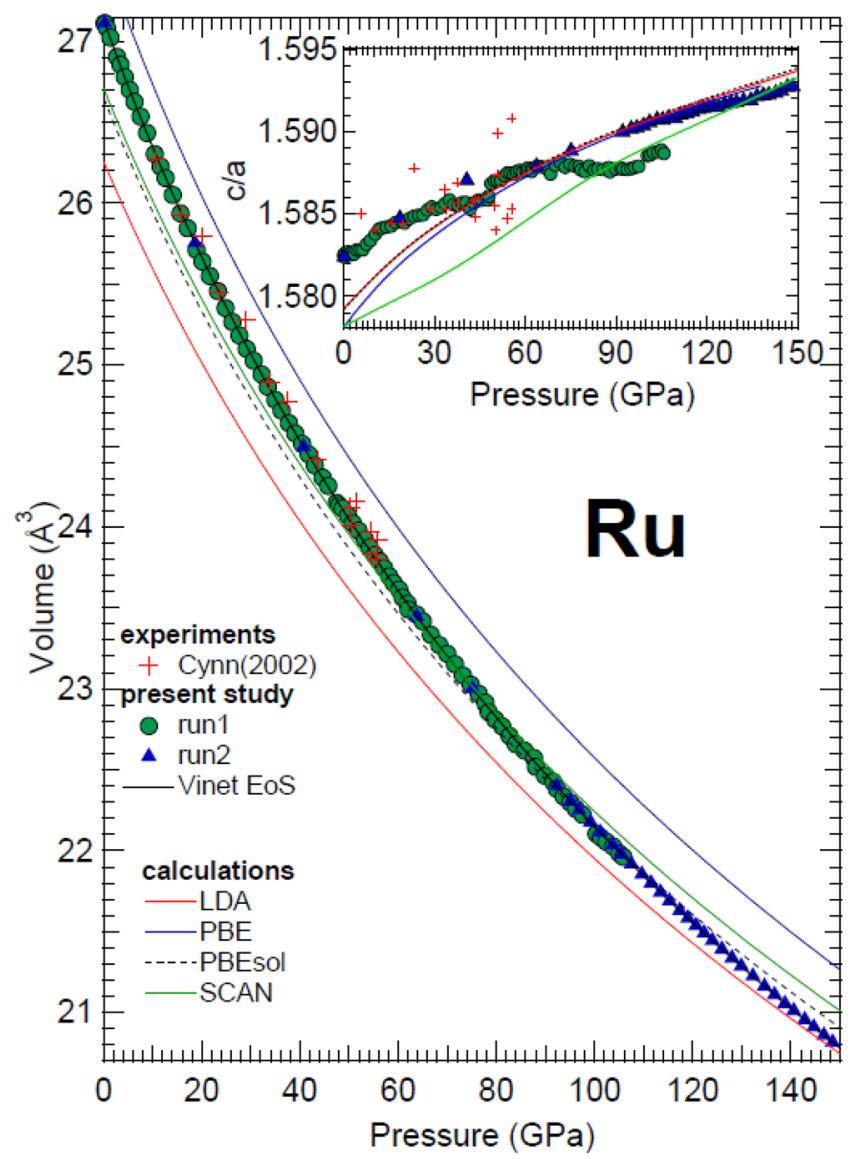

Figure 2. Measured and calculated volume of ruthenium as a function of pressure compared with literature data. Inset: evolution of the c/a ratio. Same symbols are used in both the main figure and the inset. In both figures the error bars are smaller than the used symbols.

$4 \mathrm{f}$ levels. Furthermore, the absence of anomalies in the c/a evolution in Ru rules out the presence of any other pressure-induced electronic transitions up to $150 \mathrm{GPa}$. This could be extrapolated to Technetium (Tc) as an additional $h c p$ metal with a similar electronic configuration and a similar ionic radius. Regarding the bulk modulus, it is noticeable that this parameter is very similar in $\operatorname{Re}\left(352 \mathrm{GPa}^{17}\right), \operatorname{Ir}\left(339 \mathrm{GPa}^{2}\right)$, and $\mathrm{Ru}(323 \mathrm{GPa}$ (this work)) for experiments carried out using $\mathrm{He}$ as pressure medium. Being Os surrounded by these three elements in the periodic table and having a similar electronic configuration, it is then striking that it has been reported to be the material with the lowest experimentally determined compressibility ( $462 \mathrm{GPa}^{22}$ ) having it a bulk modulus closer to $\mathrm{Re}, \mathrm{Ru}$, and $\mathrm{Ir}$ as reported from experiments performed under $\mathrm{He}\left(395 \mathrm{GPa}^{34}\right)$. 


\begin{tabular}{|l|l|l|l|l|l|l|}
\hline Reference & $\boldsymbol{V}_{\mathbf{0}}\left(\AA^{3}\right)$ & $\boldsymbol{K}_{\mathbf{0}}(\mathbf{G P a}), \boldsymbol{K}_{\mathbf{0}}^{\prime}$ & PTM & Pressure gauge & EOS & Method \\
\hline this study & $27.129(4)$ & $319.1(9), 4.40(2)$ & He & Ruby ${ }^{51}$ & Vinet & AD-XRD in DAC \\
\hline this study & $27.122(6)$ & $323.4(11), 4.150(19)$ & He & Ruby ${ }^{51}$ & BM3 & AD-XRD in DAC \\
\hline 22 & $27.148(6) *$ & $348(18), 3.30(8)$ & Ar & Ruby $^{62}$ & BM3 & ED-XRD in DAC \\
\hline this study & $26.25(2)$ & $368.89(1), 4.61(1)$ & & & BM3 & LDA \\
\hline this study & $27.53(3)$ & $314.50(3), 4.71(2)$ & & & BM3 & PBE \\
\hline this study & $26.65(1)$ & $350.46(1), 4.64(1)$ & & & BM3 & PBEsol \\
\hline this study & $26.71(1)$ & $355.36(1), 4.67(1)$ & & & BM3 & SCAN \\
\hline 22 & 26.276 & $332.8,4.3$ & & & BM3 & LDA \\
\hline
\end{tabular}

Table 3. EOS parameters of Ru measured and calculated in different experiment. The volume $V_{0}$, the bulk modulus $K_{0}$ and its pressure derivative $K_{0}^{\prime}$ are listed. Experimental methods and EOS formulation are specified. *Fixed parameter. PTM: Pressure transmitting medium. BM3: third order Birch-Murnaghan. ED-XRD: Energy dispersive X-ray diffraction.
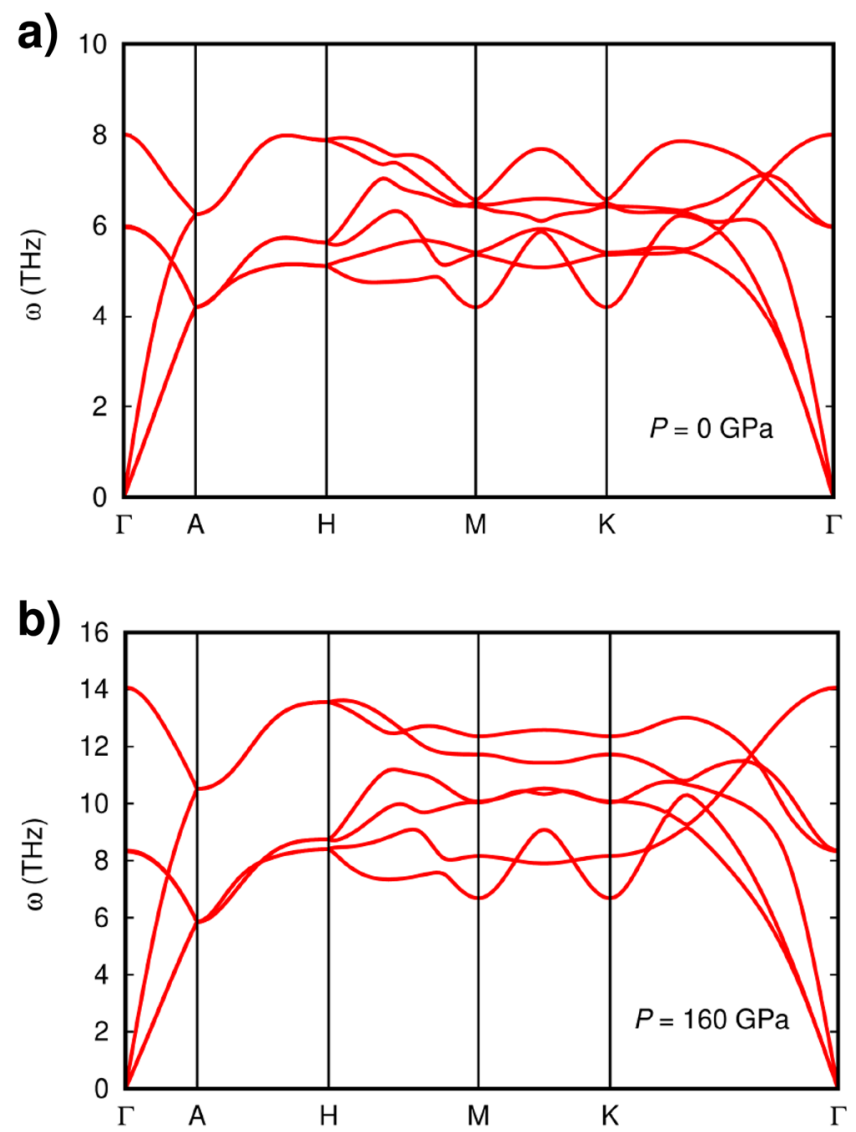

Figure 3. Simulated phononic dispersion curves of $h c p$ Ru at ambient pressure (a) and at $160 \mathrm{GPa}(\mathbf{b})$.

The compression curves and c/a evolution obtained in the present study by DFT using several exchange-correlation functionals, including $\mathrm{LDA}^{35}, \mathrm{GGA} \mathrm{PBE}^{36}$, GGA-PBEsol ${ }^{37}$, and meta-GGA SCAN ${ }^{33}$, are also reported in Fig. 2. In this case, the functional that displays an overall best agreement with the experiments is the GGA-PBEsol, hence most of the results that we present in this work are obtained with it. In particular, the difference between the bulk modulus experimentally measured and the one obtained via PBEsol functional is less than $10 \%$. Furthermore, the pressure evolution of the c/a ratio is well described by the PBEsol for pressure higher than $30 \mathrm{GPa}$, confirming the anisotropic nature of the compressed $h c p \mathrm{Ru}$. The obtained phonon dispersion at ambient and $160 \mathrm{GPa}$ are shown in Fig. 3. The dispersion at ambient pressure qualitatively agrees with that reported by Heid et al. ${ }^{38}$ In particular, at ambient pressure we observe anomalies in all branches in the vicinity of the $\mathrm{M}$ point of the Brillouin zone, with three branches being nearly degenerate and one of them being dispersionless. Our results show that $h c p$ Ru is dynamically stable up to $160 \mathrm{GPa}$. In particular, all the minima from the phonon dispersion (with the exception of the acoustic modes at Gamma) moves towards higher frequencies as pressure increase, suggesting that phonon instabilities are not expected for $h c p$ Ru even beyond $160 \mathrm{GPa}$. In addition, the anomalies around $\mathrm{M}$ are attenuated at $160 \mathrm{GPa}$ and the degeneracy between branches partially broken. 


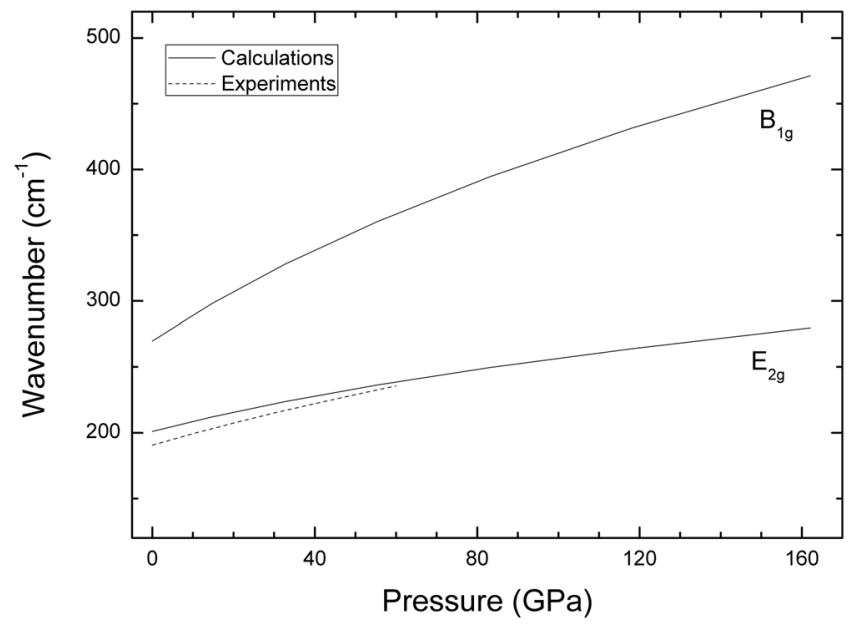

Figure 4. Pressure evolution of the Raman active $\left(E_{2 g}\right)$ and silent $\left(B_{1 g}\right)$ modes as obtained from present calculations, compared to previous work ${ }^{39}$.

The fact that the phonon dispersion at both pressures have all branches with positive frequencies, indicates that the $h c p \mathrm{Ru}$ is not only structurally, but also dynamically stable, i.e. it does not present phonon instabilities. Since the $h c p$ structure has two atoms per primitive cell, it has six possible vibrations. However, at the $\Gamma$ point, there are two pairs of degenerate modes (two optical and two acoustic branches). Based upon group-theory analysis, it is known that modes of the $h c p$ structure at $\Gamma$ are: $A_{2 u}+B_{1 g}+E_{2 g}+E_{1 u}$. The $A_{2 u}$ mode and the degenerate $E_{1 u}$ are the acoustic modes. The degenerate $E_{2 g}$ optical mode is Raman active, and the $B_{1 g}$ optical mode is a silent mode. We have calculated the pressure dependence of the optical modes which is shown in Fig. 4 . The results for the Raman-active mode agree quite well with the measurement made by Olijnyk et al. up to $60 \mathrm{GPa}^{39}$, thus they can be considered a good prediction for the pressure dependence of the Raman mode and the silent mode up to $160 \mathrm{GPa}$. The good agreement between the quasi-harmonic calculations and the experiments is an indication that anharmonic contributions are negligible in Ru at RT. Notice that the Raman active $E_{2 g}$ mode is a shear mode in which successive hexagonal planes of the $h c p$ structure beat against each other ${ }^{40}$. In contrast, the silent $B_{1 g}$ correspond to bending vibrations within the hexagonal plane ${ }^{40}$. In general, in-plane vibrational frequencies are more energetic than the out-of-plane ones. This is consistent with the results of our calculations $\left(B_{1 g}\right.$ has a higher frequency than $E_{2 g}$ ). The fact that the $E_{2 g}$ mode is associated to an inter-planar vibration along the c-axis, makes possible to link it with the $C_{44}$ elastic constant ${ }^{39}$; basically $C_{44}$ is proportional to the square power of the frequency of the $E_{2 g}$ mode. According to this hypothesis, $C_{44}$ should increase by a $60 \%$ from ambient pressure to $160 \mathrm{GPa}$, which is consistent with calculations reported by Lugovskoy et al. ${ }^{24}$ The fact that $C_{44}$ is expected to have a positive pressure derivative rules out a possible phase transition from $h c p$ to cubic structure.

High pressure - high temperature evolution. XRD experiments performed up to $56 \mathrm{GPa}$ and $960 \mathrm{~K}$ have shown that $\mathrm{Ru}$ remains in the hcp structure with no evidence of phase transitions or structural distortions. Furthermore, the obtained data do not show any formation of Ru carbides ${ }^{41}$ and/or oxides ${ }^{42}$. The pressure and temperature dependence of the Ru unit-cell obtained from four isotherms together with the RT results are shown in Fig. 5. Also results from previous high- and room-temperature studies at ambient pressure are included ${ }^{43,44}$. The results from the HT experiments carried out using $\mathrm{KCl}$ as pressure transmitting medium are consistent with both the one obtained at room temperature using He as pressure transmitting medium and the one obtained in previous studies ${ }^{43,44}$. As described before, the $R T P-V$ results are well represented by a third-order Birch-Murnaghan $\operatorname{EoS}^{45}$ with $K_{0}=323(1) \mathrm{GPa}, K_{0}^{\prime}=4.15(2)$ and $V_{0}=27.122(6) \AA^{3}$. Regarding the HT results, the isotherms shown in Fig. 5 can be described using the Birch-Murnaghan isothermal formalism ${ }^{46}$. The obtained results are shown in the figure. In the fit of the P-V-T EoS the above given values for $K_{0}, K_{0}^{\prime}$ and $V_{0}$ have been considered as fixed. The bulk modulus has been assumed to present a linear dependence from the temperature ${ }^{3}$ :

$$
K_{0}(T)=K_{0}(300)+\beta(T-300)
$$

A similar functional dependence has been assumed for the thermal expansion ${ }^{3}$ :

$$
\alpha(T)=\alpha_{0}(300)+\alpha_{1}(T-300)
$$

being $\alpha_{0}, \alpha_{1}$ and $\beta$ the only free parameters in the fit. As it can be seen in Fig. 5, the used approximations are sufficient for describing the pressure and temperature dependence of the volume in the $P$ - $T$ range explored in this study. The obtained values for the fitting parameters are $\alpha_{0}=2.2(1) 10^{-5} \mathrm{~K}^{-1}, \alpha_{1}=7.5(5) 10^{-9} \mathrm{~K}^{-2}$ and $\beta=-1.1(2) 10^{-2} \mathrm{GPa} / \mathrm{K}$. These values are comparable with those reported for the hcp phase of Ti and $\mathrm{Zn}$ in a similar temperature range ${ }^{47,48}$. The fact that a linear dependence in $T$ for the thermal expansion and bulk modulus accounts well for the experimental results up to $960 \mathrm{~K}$ indicates that Ru behaves quasi-harmonically up to this temperature. 


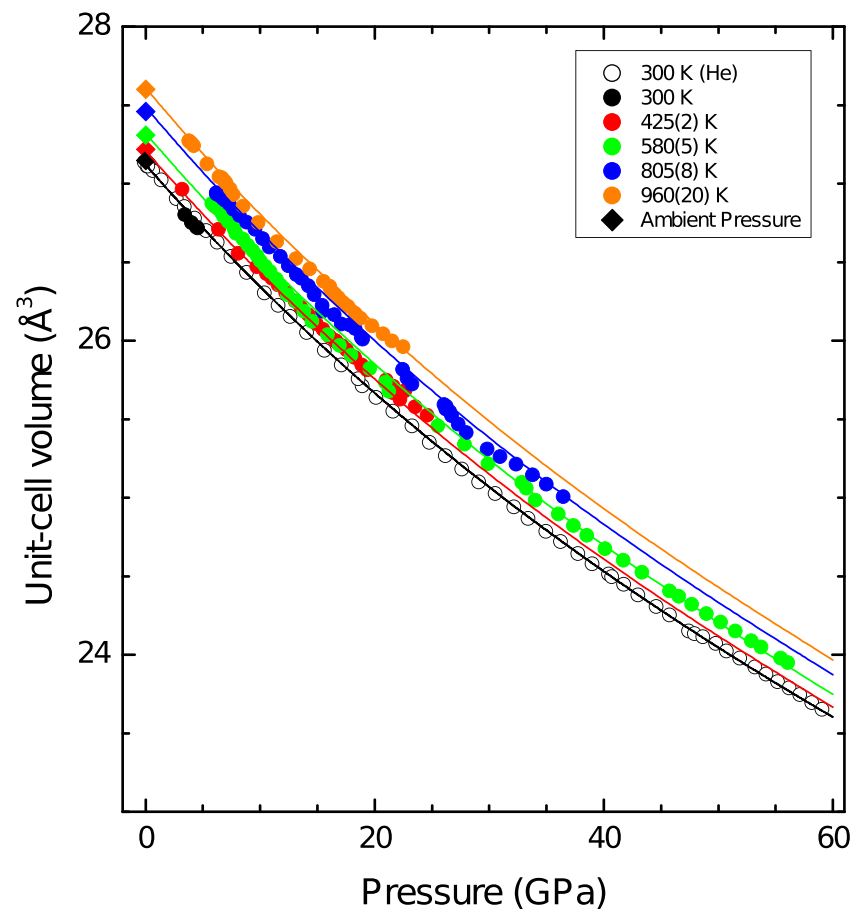

Figure 5. Compression curves of Ru obtained at different temperatures. The symbols represent the measured data whereas the lines represent the corresponding $P-V$-T EoS. The same color code has been used for both the experiments and the fits.

\section{Conclusion}

In this work the stuctural and chemical evolution of Ru has been studied under HP-HT conditions combining $\mathrm{AD}-\mathrm{XRD}$ and DAC techniques. The data have been collected along five different isotherms to a maximum of $151 \mathrm{GPa}$ at ambient temperature and $21 \mathrm{GPa}$ at $960 \mathrm{~K}$, respectively. The collected data allowed a thermal EoS to be determined following a Birch-Murnaghan formalism. In the investigated $P$ - $T$ domain, only the $h c p$ phase of Ru has been observed. The ambient temperature data have been obtained under quasi-hydrostatic conditions using $\mathrm{He}$ as pressure transmitting medium. First principle simulations based on DFT have been performed using different exchange correlation functionals to better constrain the structural and vibrational properties of $\mathrm{Ru}$ at ambient temperature. The PBEsol functional provided the best agreement with the experimental data when considering the ambient temperature EoS and the pressure dependence of the c/a ratio. The obtained vibrational modes confirm $h c p$ Ru to be structurally and dynamically stable up to at least $160 \mathrm{GPa}$.

\section{Methods}

Experimental. In this work, two series of $\mathrm{AD}-\mathrm{XRD}$ experiments were performed: one at the Materials Science and Powder Diffraction beamline BL04 at the ALBA synchrotron ${ }^{49}$, and one at the Extreme Conditions beamline I15 at the Diamond Light Source (DLS) synchrotron ${ }^{50}$. For the experiment performed at DLS, two membrane DACs were equipped with diamonds with culet sizes ranging from 200 to $100 \mu \mathrm{m}$. Whereas for the experiments performed at ALBA, four membrane DACs were equipped with diamonds with culet sizes ranging from 300 to $200 \mu \mathrm{m}$. In both cases, gaskets where prepared from pre-indented and sparkle-erosion drilled Re foils. Sample loadings were performed under an inert atmosphere to prevent sample oxidation or other possible chemical reactions. For the experiment performed at DLS, few grains of Ru (approximately $4 \mu \mathrm{m}$ each; $99.999 \%$ purity, Sigma Aldrich) were loaded at the centre of the DAC high pressure chamber. A ruby chip was placed few $\mu \mathrm{m}$ away from the sample and used as pressure gauge. Finally, the He pressure medium was loaded into the cell.

For the experiment performed at ALBA, the Ru powder was compressed into pellets using two diamond anvils. The obtained pellets were cut and loaded in the DAC high pressure chambers between two FIB-cut $\mathrm{KCl}$ disks. The $\mathrm{KCl}$ disks, acting as pressure transmitting medium as well as pressure gauges, were oven dried at $200{ }^{\circ} \mathrm{C}$ for two hours before being loaded in the DAC.

Diffraction data were collected at I15 with a monochromatic X-ray beam $(\lambda=0.4246 \AA)$ and measured using a MAR345 area detector at a distance of $300.84 \mathrm{~mm}$. During the experiment, pressure inside the high pressure chamber of the DAC was determined from the ruby luminescence method using the calibration of Dorogokupets et al. ${ }^{51}$.

At ALBA, XRD patterns were collected using an SX165 Rayonix charge-coupled device camera, with a monochromatic beam $(0.4246 \AA)$ at a sample-to-detector distance of $205.84 \mathrm{~mm}$. The DACs were heated using $240-\mathrm{V}$ Watlow coiled-cable heaters, which were wrapped around the outside of each DAC, and which can operate continuously at a power density greater than $4.65 \mathrm{~W} / \mathrm{cm}^{2}$. These heaters are capable of inducing temperatures above $900 \mathrm{~K}$ in samples ${ }^{52}$. For temperature measurements, K-type thermocouples, with an accuracy $<0.4 \%$ were 
attached to the piston of the DAC, close to one of the diamond anvils. The DAC was housed inside a dedicated water-cooled vacuum vessel which was evacuated using a rotary-backed turbo pump. An evacuated environment prevents oxidation of the diamonds at high temperatures and removes the effects of convective heating. Typically, vacuum pressures of $\sim 10^{-4}-10^{-5}$ mbar were achieved within the vessel during the course of the experiments. Water cooling ensured the vessel remained cool (approximately at room temperature (RT)) relative to the heated $\mathrm{DAC}$ and any thermally induced movement of the sample with respect to the $\mathrm{x}$-ray beam was thus minimized. This apparatus has been successfully used in previous HP-HT AD-XRD DAC experiments at different synchrotron facilities ${ }^{52-54}$.

The sample pressure was determined from the XRD patterns of $\mathrm{KCl}$ according to the thermal EOS of Dewaele et al. ${ }^{55}$.

Before each heating run, the sample was pressurized at RT to approximately $3 \mathrm{GPa}$ and then heated up while collecting XRD data. Once the target temperature was reached, isothermal compressions were performed, and XRD patterns were collected every 1-2 GPa.

In both cases, the detector geometry was calibrated with $\mathrm{a} \mathrm{LaB}_{6}$ standard using the powder calibration routine of the DIOPTAS software suite ${ }^{56}$. Masks were applied to the raw diffraction images on a per image basis before they were azimuthally integrated in DIOPTAS. The obtained diffraction data were analysed by Le Bail fitting using the routines in TOPAS software suite ${ }^{57}$, literature values for the lattice parameters of Ru were used as starting point for these refinements.

Computational methods overview. First-principles calculations based on density functional theory (DFT) have been performed to analyze the equation of state and structural and vibrational properties of $h c p$ Ru under pressure. The calculations were performed with the VASP code ${ }^{58}$ by using projector augmented-wave (PAW) method potentials ${ }^{59}$. Four different approaches have been tested in the simulations: Local-density approximation (LDA), generalized-gradient approximation with Perdew-Burke-Ernzerhof (GGA-PBE) and Perdew-Burke-Ernzerhof for solids (GGA-PBEsol) functionals, and meta-GGA with strongly constrained and appropriately normed semilocal functionals (SCAN). The electronic states $4 p^{6} 5 s^{1} 4 d^{7}$ are considered as valence. Wave functions are represented in a plane-wave basis truncated at $650 \mathrm{eV}$. By using these parameters and dense k-point grids of $16 \times 16 \times 12$ for integration within the first Brillouin zone (IBZ), energies are converged to within $1 \mathrm{meV}$ per formula unit. In the geometry relaxations, a tolerance of $0.01 \mathrm{eV} . \AA^{-1}$ is imposed in the atomic forces. $A b$ initio phonon frequencies are calculated with the small-displacement $\operatorname{method}^{25,60}$ in order to assess the vibrational stability of highly compressed $h c p$ Ru and estimate the P-dependence of the corresponding Raman mode. In the small-displacement approach, the force-constant matrix is calculated in real-space by considering the proportionality between atomic displacements and forces. The quantities with respect to which the phonon calculations are converged include the size of the supercell, size of the atomic displacements, and numerical accuracy in the sampling of the IBZ. The settings providing a quasi-harmonic free energies converging to within $5 \mathrm{meV}$ per formula unit are the following: $4 \times 4 \times 3$ supercells (where the figures indicate the number of replicas of the unit cell along the corresponding lattice vectors), atomic displacements of $0.02 \AA$, and k-point grids of $4 \times 4 \times 4$. The value of the phonon frequencies are obtained with the PHON code developed by Alfé ${ }^{61}$. In using this code, the translational invariance of the system is exploited to impose the three acoustic branches to be exactly zero at the center of the Brillouin zone, and apply central differences in the atomic forces.

\section{Data Availability}

The datasets generated during and/or analysed during the current study are available from the corresponding author on reasonable request.

\section{References}

1. Tal, A. et al. Pressure-induced crossing of the core levels in 5d metals. Phys. Rev. B 93, 205150 (2016).

2. Monteseguro, V. et al. Phase stability and electronic structure of iridium metal at the megabar range. Sci. Reports 9, 1 (2019).

3. Anzellini, S. et al. In situ characterization of the high pressure - high temperature melting curve of platinum. Sci. Reports $\mathbf{9}, 13034$, https://doi.org/10.1038/s41598-019-49676-y (2019).

4. Hunt, L. \& Lever, F. Platinum metals: A survey of productive resources to industrial uses. Platinum Met. Rev. 13, 126 (1969).

5. Tonkov, E. \& Ponyatovsky, E. Phase Transformation of elements under high pressure - Advances in metallic alloys (CRC press, 2005).

6. Rao, C. \& Trivedi, D. Chemical and electrochemical deposition of platinum group metals and their applications. Coord. Chem. Rev. 249, 613 (2005).

7. Raub, C. Superconductivity of the platinum metals and their alloys. Mater. Des. 5, 129 (1984).

8. Thirunavukkarasu, V., Kozhushkov, S. \& Ackermann, L. C-h nitrogenation and oxygenation by ruthenium catalysis. Chem Comm 50, 29 (2014).

9. Miao, S. et al. Ru nanoparticles immobilized on montmorillonite by ionic liquids: a highly efficient heterogeneous catalyst for the hydrogenation of benzene. Angewandte Chemie, Int. Ed. 45, 266 (2005).

10. Nguyen, S., Grubbs, R. \& Ziller, J. Syntheses and activities of new single-component, ruthenium-based olefin metathesis catalyst. J. Am. Chem. Soc. 115, 9858 (1993).

11. Zhang, Y. et al. Diverse ruthenium nitrides stabilized under pressure: a theoretical prediction. Sci. Reports 6, 33506 (2016).

12. Niwa, K. et al. Discovery of the last remaining binary platinum-group pernitride run2. Chem. 20, 13885 (2014).

13. Sun, W., Chakraborty, S., Koepernik, K. \& Ahuja, R. Stabilizing a novel hexagonal ru2c through lifshitz transition under pressure. arXiv preprint arXiv:1310.0170 (2013).

14. Huang, X. et al. Thermal equation of state of molybdenum determined from in situ synchrotron $\mathrm{x}$-ray diffraction with laser-heated diamond anvil cells. Sci. Reports 6, 19923 (2016).

15. Santamaria-Perez, D. et al. X-ray diffraction measurements of mo melting to $119 \mathrm{gpa}$ and the high pressure phase diagram. J. Chem. Phys. 130, 124509 (2009).

16. Hrubiak, R., Meng, Y. \& Shen, G. Microstrdiagram define melting of molybdenum at high pressure. Nat. Commun. 8, 14562 (2017).

17. Anzellini, S., Dewaele, A., Occelli, F., Loubeyre, P. \& Mezouar, M. Equation of state of rhenium and application for ultra high pressure calibration. J. Appl. Phys. 115, 043511 (2014). 
18. Dubrovinsky, L., Dubrovinskaia, N., Prakapenka, V. \& Abakumov, A. Impcalibration of micro-ball nanodiamond anvils for highpressure studies above 6 mbar. Nat. Commun. 3, 1163 (2012).

19. Bucher, E., Muller, J., Olsen, J. \& Palmy, C. Superconductivity of osmium and ruthenium under pressure. Cryog. 5, 283 (1965).

20. Clendenen, R. \& Drickamer, H. The effect of pressure on the volume and lattice parameters of ruthenium and iron. J. Phys. Chem. Solids 25, 865 (1964).

21. Perez-Albuerne, E., Forsgren, K. \& H. G. Apparatus for x-ray measurements at very high pressure. Rev. Scie 35, 29 (1964).

22. Cynn, H., Klepeis, J., Yoo, C. \& D. A. Osmium has the lowest experimentally determined compressibility. Phys. Rev. Lett. 88 (2001).

23. Blackburn, L., Kaufman, L. \& Cohen, M. Phase transformation in iron-ruthenium alloys under high pressure. ACTA Metall. 13, 533 (1965).

24. Longovskoy, A., Belov, M., Krasilnikov, O. \& Vekilov, Y. Ruthenium of the hcp ruthenium at high ppressure from first principle. J. Appl. Phy 116, 103507 (2014).

25. Cazorla, C., Alfe, D. \& Gillan, M. Zero-temperature generalized phase diagram of the $4 \mathrm{~d}$ transition metals under pressure. Phys. Rev. $B$ 77, 224103 (2008).

26. Takemura, K. Evaluation of the hydrostaticity of a helium-pressure medium with powder x-ray diffraction techniques. J. Appl. P 89, $662(2001)$.

27. Williamson, G. \& Hall, W. X-ray line broadening from filed aluminium and wolfram. ACTA Metall. 1, 22 (1953).

28. Dewaele, A. \& Loubeyre, P. Pressurizing conditions in helium-pressure-transmitting medium. High Press. Res. 27, 419 (2007).

29. Vinet, P., Ferrante, J., Rose, J. \& Smith, J. Compressibility of solids. J. Geophys. Res. 92, 9319 (1987).

30. Angel, R., Gonzalez-Platas, J. \& Alvaro, M. Eosfit7c and a fortran module (library) for equation of state calculations. Z. Kristallogr. 229, 405 (2014)

31. Cohen, R. E., Stixrude, L. \& Wasserman, E. Tight-binding computations of elastic anysotropy of fe, xe, and si under compression. Phys. Rev. B 56, 8575 (1997).

32. Dubrovinsky, L. et al. The most incompressible metal osmium at static pressures above 750 gigapascals. Nat. 525, 226 (2015).

33. Sun, J., Ruzsinszky, A. R. \& Perdew, J. Strongly constrained and appropriately normed semilocal density functional. Phys. Rev. Lett. 115, $036402(2015)$.

34. Takemura, K. Bulk modulus of osmium: High-pressure powder $\mathrm{x}$-ray diffraction experiments under quasihydrostatic conditions. Phys. review B 70, 012101 (2004).

35. Caperley, D. \& Alder, B. Ground state of the electron gas by a stochastic method. Phys. Rev. Lett. 45, 556 (1980).

36. Perdew, J., Burke, K. \& Ernzerhof, M. Generalized gradient approximation made simple. Phys. Rev. Lett. 77, 3865 (1996).

37. Perdew, J. et al. Restoring the density-gradient expansion for exchange in solid surfaces. Phys. Rev. Lett. 100, 136406 (2008).

38. Heid, R., Pintschovius, L., Reichardt, W. \& Bohnen, K. Anomalous lattice dynamics of ruthenium. Phys. review B 61, 12059 (2000).

39. Olijnyk, H., Jephcoat, A. \& Refson, K. On optical phonons and elasticity in the hcp transition metals fe, ru and re at high pressure. Europhys. Lett. 53, 504 (2001).

40. Cazorla, C., Errandonea, D. \& Sola, E. High-pressure pphase, vibrational properties, and electronic structure of ne(he) ${ }_{2}$ and ar(he) $)_{2}$ : A first-principle study. Phys. Rev. B 80, 064105 (2009).

41. Zhao, Z. et al. Carbon coated face-centered cubic ru-c nanoalloys. Nanoscales 6, 10370 (2014).

42. Chan, H., Takoudis, C. \& Weaver, M. High-pressure oxidation of ruthenium as probed by surface-enhanced raman and x-ray photoelectron spectroscopies. J. Catal. 172, 336 (1997)

43. Cernohorsky, M. The precision determination of lattice parameters. Acta Crystallogr. 13, 823 (1960).

44. Owen, E. \& Roberts, E. The crystal parameters of osmium and ruthenium at different temperatures. Zietschr. Kristallographie 96, 497 (1937).

45. Birch, F. Elasticity and constitution of the earth's interior. J. Geophys. Res. 57, 227 (1952).

46. Errandonea, D., Meng, Y., Hausermann, D. \& Uchida, T. Study of the phase transformation and equation of state of magnesium by synchrotron x-ray diffraction. J. Phys.: Condens. Matter 15, 1277 (2003).

47. Zhang, J. et al. Thermal equation of state for titanium obtained by high pressure - temperature diffraction studies. Phys. Rev. B 78, 054119 (2008).

48. Errandonea, D. et al. High-pressure/ high-temperature phase diagram of zinc. J. Phys.: Condens. Matter 30, 295402 (2018).

49. Fauth, F., Peral, I. \& Knapp, C. P. P. The new material science powder diffraction beamline at alba synchrotron. Powder Diffr. 28, S360 (2013).

50. Anzellini, S. et al. Laser-heating system for high-pressure x-ray diffraction at the extreme conditions beamline 115 at diamond light source. J. Synchrotron Radiat. 25, 1 (2018).

51. Dorogokupets, P. \& Dewaele, A. Equation of state of mgo, au, pt, nacl-b1, and nacl-b2: Internally consistent hightemperature pressure scales. High Press. 27, 431 (2007).

52. Stinton, G. et al. Equation of state and high-pressure/high-temperature phase diagram of magnesium. Phys. Rev. B 90, 134105 (2014).

53. Cazorla, C. et al. Thallium under extreme compression. J. Phys.: Condens. Matter 28, 445401 (2016).

54. Anzellini, S. et al. Phase diagram of calcium at high pressure and high temperature. Phys. Rev. Mater. 2, 083608 (2018).

55. Dewaele, A. et al. High-pressure-high-temperature equation of state of kcl and kbr. Phys. Rev. B 85, 214105 (2012).

56. Prescher, C. \& Prakapenka, V. Dioptas: a program for reduction of two-dimensional $\mathrm{x}$-ray diffraction data and data exploration. High Press. 35, 223 (2015).

57. Coelho, A. Topas and topas-academic: an optimization program integrating computer algebra and crystallography objects in $\mathrm{c}++$. J. Applyed Crystallogr. 51, 210 (2018).

58. Kresse, G. \& Furthmüller, J. Efficient iterative schemes for ab initio total-energy calculations using a plane-wave basis set. Phys. Rev. B 54, 11169 (1996).

59. Bloch, P. Projector augmented-wave method. Phys. Rev. B 50, 17953 (1994).

60. Cazorla, C. \& Boronat, J. Simulation and understanding of atomic and molecular quantum crystals. Rev. Mod. Phys. 89, 035003 (2017).

61. Alfè, D. Phon: A program to calculate phonons using the small displacement method. Comp. Phys. Commun. 180, 2622 (2009).

62. Mao, H., Bell, P., Shaner, J. \& Steinberg, D. Specific volume measurements of cu, mo, pd, and ag and calibration of the ruby $\mathrm{r} 1$ fluorescence pressure gauge from 0.06 to 1 mbar. J. Appl. Phys. 49, 3276 (1978).

\section{Acknowledgements}

The authors acknowledge the DLS and ALBA synchrotron facilities for provision of beamtime on the beamlines I15 and BL04, respectively (DLS ref. NT21190, ALBA ref. 2018082951). We also acknowledge the support from the Natural Environment Research Council of Great Britain and Northern Ireland via grants NE/M000117/1 and NE/M00046X/1. E.B. and D.E. are thankful for the financial support to this research from the Spanish Ministerio de Ciencia, Innovación y Universidades, the Spanish Research Agency, and the European Fund for Regional Development under Grant No. MAT2016-75586-C4-1-P and by Generalitat Valenciana through the grant Prometeo/2018/123 EFIMAT and ValI+D, APOSTD/2017/075. C.P. acknowledges the Spanish Mineco Project 
FIS2017-83295-P. S.B. has received founding from the European Research Council (ERC) under the European Union's Horizon 2020 research and innovation program (grant agreement No 670787). (c)British Crown Owned Copyright 2019/AWE. Published with permission of the Controller of Her Britannic Majesty's Stationery Office.

\section{Author Contributions}

S.A. and D.E. conceived the experiment(s). S.A., D.E., S.M., V.M., S.B., E.B., D.D.A. and C.P. conducted the experiment(s). S.A. and D.E. analyzed the results. C.C. performed the theoretical calculations. The manuscript is written through contributions of all authors. All authors have given approval to the final version of the manuscript.

\section{Additional Information}

Competing Interests: The authors declare no competing interests.

Publisher's note Springer Nature remains neutral with regard to jurisdictional claims in published maps and institutional affiliations.

(c) (i) Open Access This article is licensed under a Creative Commons Attribution 4.0 International License, which permits use, sharing, adaptation, distribution and reproduction in any medium or format, as long as you give appropriate credit to the original author(s) and the source, provide a link to the Creative Commons license, and indicate if changes were made. The images or other third party material in this article are included in the article's Creative Commons license, unless indicated otherwise in a credit line to the material. If material is not included in the article's Creative Commons license and your intended use is not permitted by statutory regulation or exceeds the permitted use, you will need to obtain permission directly from the copyright holder. To view a copy of this license, visit http://creativecommons.org/licenses/by/4.0/.

(C) The Author(s) 2019 